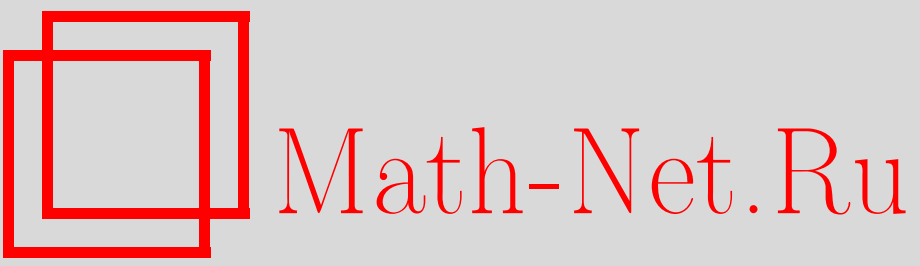

В. В. Стружанов, Свойства разупрочняющихся материалов и определяющие соотношения при одноосном напряженном состоянии, Вестн. Сам. гос. техн. ун-та. Сер. Физ.-мат. науки, 2007, выпуск 2(), 69-78

DOI: https://doi.org/10.14498/vsgtu532

Использование Общероссийского математического портала Math-Net.Ru подразумевает, что вы прочитали и согласны с пользовательским соглашением

http: //www . mathnet.ru/rus/agreement

Параметры загрузки:

IP : 3.85 .5 .30

26 апреля 2023 г., 13:30:16 
Вестн. Сам. гос. техн. ун-та. Сер.: Физ.-мат. науки. - 2007. - № 2 (15). - С. 69-78. - ISSN 1991-8615

УДК 539.3

\section{В. В. Стружанов}

\section{СВОЙСТВА РАЗУПРОЧНЯЮЩИХСЯ МАТЕРИАЛОВ И ОПРЕДЕЛЯЮЩИЕ СООТНОШЕНИЯ ПРИ ОДНООСНОМ НАПРЯЖЁННОМ СОСТОЯНИИ}

На основании результатов, полученных при исследовании модифицированной модели Мазинга, позволяющей прогнозировать наличие падающей ветви на диагралме дебормирования, выделены три класса материалов, а именно, упруго хрупкие, упругопластические и партипластические материаль. Для каждого класса получены определяющие соотношения при одноосном напряжённо-дебормированном состоянии.

1. Идеализация свойств материала. Экспериментальные данные $[1,2]$, модифицированная модель Мазинга [3] и другие структурные модели [4] указывают на то, что наиболее полно свойства материалов при растяжении определяет так называемая полная диаграмма деформирования $\sigma(\varepsilon)$, состоящая из восходящей и ниспадающей до нуля ветвей (кривые 1 на рис. $1-3)$. Характерные точки диаграммы: $\sigma^{t}, \sigma^{B}, \varepsilon^{t}, \varepsilon^{B}$ - соответственно предел пропорциональности, предел прочности и отвечающие им продольные деформации растяжения, $\varepsilon^{Z}$ - предельная продольная деформация. Отметим, что в частном случае предел пропорциональности может совпадать с пределом текучести $\sigma^{T}$ (тогда $\varepsilon^{T}$ - деформация начала текучести).

Важной характеристикой материала является инкрементальный (мгновенный, касательный) продольный модуль $E^{p}=\frac{d \sigma}{d \varepsilon}$. На стадии упругости $E^{p}=E$ ( $E-$ модуль Юнга). Когда $E^{p}>0$, то материал находится в состоянии упрочнения (рост деформации растяжения сопровождается возрастанием сопротивления материала), при $E^{p}<0-$ в состоянии разупрочнения (рост деформации сопровождается падением сопротивления материала). Очевидно, что

$$
\sigma(\varepsilon)=\int_{0}^{\varepsilon} E^{p}(\varepsilon) d \varepsilon, \quad \int_{0}^{\varepsilon^{Z}} E^{p}(\varepsilon) d \varepsilon=0 .
$$

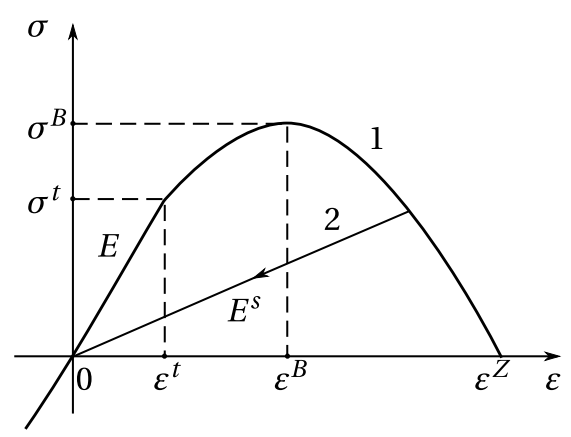

Рис. 1. Модель упруго хрупкого материала

Отсюда если даже функция $E^{p}$ в некоторой точке имеет разрыв второго рода (обращается в бесконечность), данные интегралы существуют.

Отклонение диаграммы деформирования от начального прямолинейного (упругого) участка объясняется тем, что диссипативные процессы уже начинают играть существенную роль на макроуровне (исключение составляют нелинейно упругие материалы). После нагружения в начальный момент в материале возникают напряжения, связанные с деформацией линейным законом упругости. Затем материал стремится снизить уровень напряжений. Для этого существуют два механизма, а именно, пластическая деформация (механическая диссипация) и нарушение сплошности (континуальное разрушение). В результате за малый промежуток времени происходит релаксация напряжений. Если явно

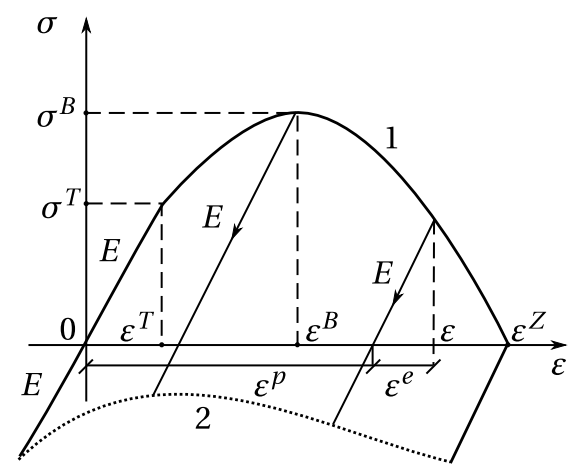

Рис. 2. Модель упругопластического материала не проявляются эффекты ползучести, то время релаксации меньше, чем скорость нагружения.

Известно [5], что при активном деформировании практически все материалы неотличимы от нелинейно упругих материалов. Различия проявляются только при разгрузке. Следовательно, только по разгрузке можно определить характер диссипативного процесса. На качественном уровне можно выделить три большие группы материалов, а именно, упруго хрупкие (диссипация посредством континуального разрушения), упругопластические (диссипация посредством пластической деформации) и партипластические (частично пластические) материалы (имеют место оба вида диссипации).

Проводя идеализацию характеристик разгрузки, полученных при исследовании модифицированной модели Мазинга [3], определим качественные (отличительные) особенности разгрузки для каждого типа материала. 


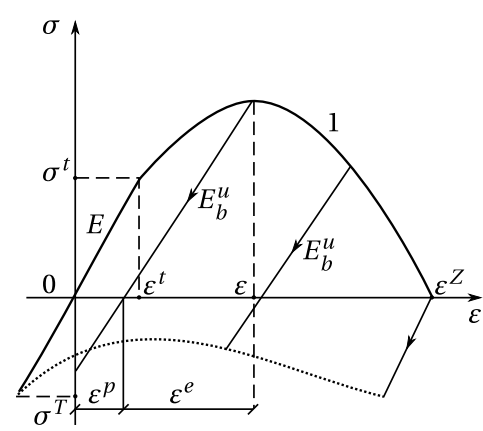

a

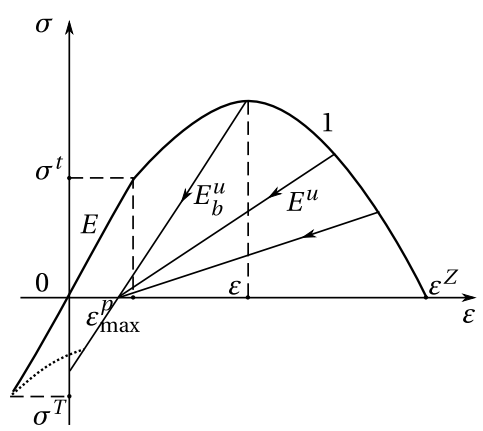

б

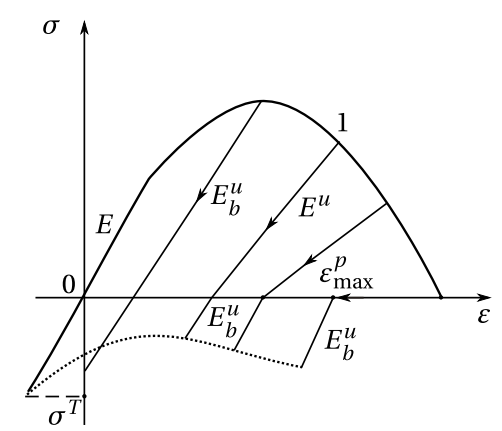

$B$

Рис. 3. Модель партипластического материала с различными вариантами разгрузки

Упруго хрупкий материал состоит из абсолютно хрупких элементов. При деформировании с определённого момента (конца упругой стадии) в нем начинается множественное трещинообразование без всяких следов пластической деформации. Следовательно, упруго хрупкий материал диссипирует подводимую энергию только посредством механизма континуального разрушения. Разгрузка происходит по секущей линии (прямая 2, рис. 1) без образования остаточных деформаций. Модуль разгрузки $E^{u}$ равен секущему модулю $E^{s}=\frac{\sigma}{\varepsilon}$. При последующем после разгрузки сжатии, когда закроются все дефекты, материал ведёт себя упруго и модуль упругости равен E. Таким образом, после разгрузки материал приобретает свойство разномодульности, т.е. он по-разному сопротивляется растяжению и сжатию на упругой стадии деформирования.

Упругопластическая модель с разупрочнением основана на распространении допущений классической модели упругопластического материала на стадию разупрочнения. Напомним, что в этой модели разгрузка происходит по линейному закону и модуль разгрузки равен модулю продольной упругости $E$, т. е. пренебрегается незначительным падением модуля разгрузки, происходящим вследствие пластического разрыхления материала [6].

Исследование модифицированной модели Мазинга [3] показывает, что падающая ветвь диаграммы деформирования появляется в результате разрушения элементов системы. (Этот вывод присутствует и в других работах, например, в книге [7]). Если система состоит из упругоидеальнопластических элементов, то на восходящей ветви (при отсутствии разрушений) данная структурная модель соответствует классической модели. На падающей же ветви наблюдается «выполаживание» (монотонное убывание) модуля разгрузки, которое происходит из-за разрушения части упруго-идеальнопластических элементов после завершения процесса пластического течения (исчерпания пластичности). Кроме того, прямая разгрузки искривляется, что вызвано постепенным закрытием дефектов и включением в работу частей разрушенных элементов.

Однако, если плотность распределения деформаций разрушения по элементам системы имеет значительную асимметрию в сторону больших величин деформаций разрушения, то существенное «выполаживание» модуля разгрузки и нарушение линейного характера разгрузки будет происходить уже в самом конце процесса деформирования. Поэтому для таких материалов правомерно распространение допущений классической модели и на стадию разупрочнения.

Таким образом, идеализированная модель упругопластического материала предполагает, что разгрузка из любой точки полной диаграммы, в том числе и с падающей ветви, происходит по прямой, параллельной начальному упругому участку диаграммы, и модуль разгрузки $E^{u}=E$ (рис. 2). Отсюда материал диссипирует подводимую энергию только посредством пластической деформации (механическая диссипация). Полная деформация в таком материале однозначно представляется суммой $\varepsilon=\varepsilon^{e}+\varepsilon^{p}$, где $\varepsilon^{e}=\frac{\sigma}{E}-$ упругая составляющая, $\varepsilon^{p}-$ пластическая составляющая полной деформации (рис. 2). После разгрузки материал обладает остаточной деформацией, равной $\varepsilon^{p}$.

И, наконец, последующее после разгрузки сжатие происходит по тому же закону, что и разгрузка, причём имеет место как классический эффект Баушингера (при сжатии после разгрузки с восходящей ветви диаграммы растяжения предел текучести уменьшается), так и инверсия этого эффректа, заключающаяся в том, что предел текучести при разгрузке с падающей ветви и последующем сжатии возрастает. На рис. 2 кривая пределов текучести при сжатии показана пунктирной линией 2 . 
Более общей является модель партипластического материала, синтезирующая упруго хрупкую и упругопластическую модели. Она описывает свойства материалов, состоящих из хрупких и пластических элементов. При деформировании такого материала действуют оба механизма диссипации (механическая диссипация и континуальное разрушение). В этой модели разгрузка также происходит по линейному закону, но модуль разгрузки $E^{u}<E$ и уменьшается с ростом продольной деформации (рис. 3). Этот процесс связан с континуальным разрушением. Полная деформация однозначно представляется суммой $\varepsilon=\varepsilon^{e}+\varepsilon^{p}$, где $\varepsilon^{e}=\frac{\sigma}{E^{u}}$ (рис. $\left.3, a\right)$. Возникновение пластической составляющей $\varepsilon^{p}$ обусловлено механической диссипацией.

Деформации партипластического материала достаточно велики. При этом в хрупких элементах возникает столь большие напряжения, что они разрушаются ещё до разрушения всего элементарного макрообъёма материала. В результате этого процесса модуль разгрузки уменьшается до некоторой величины $E_{b}^{u}$ (рис. 3). Затем, если пластические элементы не разрушаются, он сохраняет данное значение.

В модифицированной модели Мазинга [3] после начала разрушения пластичных элементов на диаграмме деформирования появляется падающая ветвь, разгрузка с которой представляется ломанной линией. Сначала модуль разгрузки ещё больше уменьшается вследствие разрушения части пластических элементов, а затем, после того как дефекты в разрушенных пластичных элементах закроются, он принимает значение, имевшее место до начала разрушения этих элементов. Исходя из этого фракта выделим три идеалиализированных случая. В первом случае модуль разгрузки с падающей ветви постоянен и равен $E_{b}^{u}$. При последующем сжатии этот модуль сохраняется (рис. 3, а). Таким образом, материал приобретает свойства, характерные для идеализированной упругопластической модели. Во втором случае модуль разгрузки уменьшается до нуля, и при этом не возникает новых пластических деформаций. То есть произошло охрупчивание всех пластических элементов, и материал приобрёл свойства, характерные для упругопластической модели. Разгрузка происходит до деформации, равной максимальному значению пластической деформации, достигнутой до охрупчивания, и дальнейшее сжатие происходит с модулем $E_{b}^{u}$ (рис. 3 , б). В третьем случае считаем, что модуль разгрузки имеет некоторое промежуточное значение между первым и вторым вариантами, и он убывает от значения $E_{b}^{u}$ до нуля (рис. 3, в). Модуль последующего после разгрузки сжатия равен значению $E_{b}^{u}$ (после разгрузки закрываются все дефекты в пластичных элементах).

И, наконец, в первом и третьем случаях имеет место инверсия эффректа Баушингера. То есть сначала предел текучести при сжатии уменьшается, а затем возрастает. Кривые пределов текучести при сжатии показаны на рис. 3, а, в пунктирной линией. Во втором случае предел текучести при сжатии убывает до некоторого значения и затем не изменяется (пунктирная кривая на рис. 3 , б).

2. Определяющие соотношения. Рассмотрим сначала общий случай партипластического тела. Запишем уравнение второго закона термодинамики с учётом необратимости и изотермичности процесса деформирования в виде [8]

$$
d F=\frac{1}{\rho} \sigma d \varepsilon-d g
$$

где $F$ - свободная энергия, $\rho$ - плотность материала, $\sigma d \varepsilon-$ элементарная работа напряжения растяжения, $d g \geqslant 0$ - некомпенсированное тепло (диссипация). Параметрами термодинамического состояния являются $\varepsilon^{e}, \varepsilon^{p}$ и параметр $\omega$, характеризующий поврежденность материала. Полагаем, что упругие свойства материала не зависят от пластических деформаций, т. е. $F=F\left(\varepsilon^{e}, \omega\right)$. Тогда, учитывая равенство $d \varepsilon=d \varepsilon^{e}+d \varepsilon^{p}$, имеем

$$
\frac{\partial F}{\partial \varepsilon^{e}} d \varepsilon^{e}+\frac{\partial F}{\partial \omega} d \omega=\frac{1}{\rho} \sigma\left(d \varepsilon^{e}+d \varepsilon^{p}\right)-d g \quad \text { или } \quad\left(\frac{\partial F}{\partial \varepsilon^{e}}+\frac{\sigma}{\rho}\right) d \varepsilon^{e}+\left(\frac{\partial F}{\partial \omega} d \omega-\frac{\sigma}{\rho} d \varepsilon^{p}+d g\right)=0 .
$$

Отсюда [8]

$$
\begin{gathered}
\sigma=\rho \frac{\partial F}{\partial \varepsilon^{e}} \\
\rho d g=\sigma d \varepsilon^{p}-\rho \frac{\partial F}{\partial \omega} d \omega .
\end{gathered}
$$

Из (2) следует, что $\rho d g=d g^{p}+d g^{\omega}$, где

$$
d g^{p}=\sigma d \varepsilon^{p}
$$


- механическая диссипация,

$$
d g^{\omega}=-\rho \frac{\partial F}{\partial \omega} d \omega
$$

- диссипация континуального разрушения.

Функция свободной энергии определяет ту часть внутренней энергии, которая остаётся в теле и не переходит в теплоту. Для рассматриваемого процесса деформирования она равна

$$
F=\frac{1}{\rho}\left(\frac{1}{2} E \varepsilon^{e} \varepsilon^{e}-\frac{1}{2} \omega E \varepsilon^{e} \varepsilon^{e}\right),
$$

где первый член в скобках является потенциальной энергией упругих деформаций после механической диссипации (релаксации напряжений вследствие пластической деформации) а второй член - энергией, потерянной из-за континуального разрушения (релаксации напряжений вследствие хрупких микроразрушений). Тогда, используя фрормулу (1), получаем

$$
\sigma=E \varepsilon^{e}-\omega E \varepsilon^{e}=E(1-\omega) \varepsilon^{e}=E(1-\omega)\left(\varepsilon-\varepsilon^{p}\right) .
$$

Здесь $E(1-\omega)=E^{u}-$ модуль разгрузки.

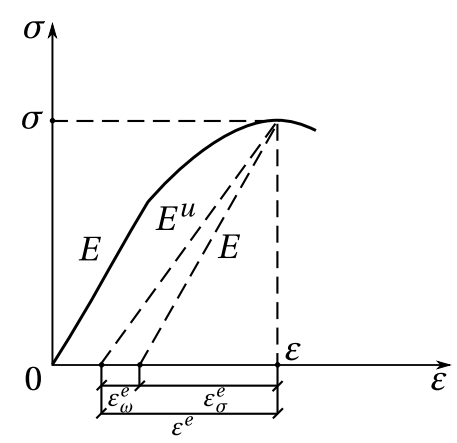

Рис. 4. Составляющие упругой деформации

Подставляя затем фрунццию $F$ в выражение (4), находим

$$
d g^{\omega}=\frac{1}{2} E \varepsilon^{e} \varepsilon^{e} d \omega
$$

Наконец из соотношения (5) следует, что $\varepsilon^{e}=\frac{\sigma}{E}+\omega \varepsilon^{e}=\varepsilon_{\sigma}^{e}+\varepsilon_{\omega}^{e}$ (см. рис. 4). Так как $\sigma=E^{u} \varepsilon^{e}$, то $\varepsilon^{e}=\frac{E^{u}}{E} \varepsilon^{e}+\omega \varepsilon^{e}$ и тогда

$$
\omega=1-\frac{E^{u}}{E}
$$

Отсюда

$$
d \omega=-\frac{d E^{u}}{E} .
$$

Далее, используя формулу (5), находим

$$
d \sigma=E(1-\omega)\left(d \varepsilon-d \varepsilon^{p}\right)-E\left(\varepsilon-\varepsilon^{p}\right) d \omega .
$$

С другой стороны, справедливо инкрементальное соотношение

$$
d \sigma=E^{p} d \varepsilon
$$

Приравнивая выражения (9) и (10), получаем дифференциальное уравнение

$$
\left(\varepsilon-\varepsilon^{p}\right) \frac{d \omega}{d \varepsilon}+\omega\left(1-\frac{d \varepsilon^{p}}{d \varepsilon}\right)=1-\frac{E^{p}}{E}-\frac{d \varepsilon^{p}}{d \varepsilon}
$$

с начальными условиями

$$
\omega(0)=0, \quad \varepsilon^{p}(0)=0, \quad E^{p}(0)=E .
$$

Преобразуя уравнение (11) с учётом равенства (8), имеем

$$
\frac{d \varepsilon^{p}}{d \varepsilon}=1-\frac{E^{p}}{E^{u}}+\frac{\left(\varepsilon-\varepsilon^{p}\right)}{E^{u}} \frac{d E^{u}}{d \varepsilon} .
$$

Уравнение (11) (или (12)) определяет кинетику совместного формирования повреждённости и пластической деформации.

Если повреждённость $\omega$ во все время деформирования равна нулю (упругопластический материал), то определяющее соотношение (5) принимает вид

$$
\sigma=E\left(\varepsilon-\varepsilon^{p}\right)
$$


а кинетическое уравнение (11) преобразуется в уравнение

$$
\frac{d \varepsilon^{p}}{d \varepsilon}=1-\frac{E^{p}}{E} \text {. }
$$

Уравнение (14) определяет кинетику развития пластических деформаций при отсутствии повреждённости.

Когда во все время деформирования отсутствует пластическая деформация (упруго хрупкий материал), то соответственно имеем

$$
\begin{gathered}
\sigma=E(1-\omega) \varepsilon=E^{s} \varepsilon, \\
\varepsilon \frac{d \omega}{d \varepsilon}+\omega=1-\frac{E^{p}}{E} \quad\left(\frac{d(\varepsilon \omega)}{d \varepsilon}=1-\frac{E^{p}}{E}\right) .
\end{gathered}
$$

Уравнение (16) определяет кинетику развития повреждённости при отсутствии пластической деформации.

Непосредственно проверяется, что выражение (7) является общим решением дифференциального уравнения (11) (а также и уравнения (16) при $E^{u}=E^{s}$ ). Действительно, имеем

$$
\begin{aligned}
\frac{d \omega}{d \varepsilon}=-\frac{1}{E} \frac{d E^{u}}{d \varepsilon}=-\frac{1}{E} \frac{d}{d \varepsilon}\left(\frac{\sigma}{\varepsilon-\varepsilon^{p}}\right)=-\frac{1}{E} \frac{\left(\varepsilon-\varepsilon^{p}\right) \frac{d \sigma}{d \varepsilon}-\sigma\left(1-\frac{d \varepsilon^{p}}{d \varepsilon}\right)}{\left(\varepsilon-\varepsilon^{p}\right)^{2}}= & \\
& =-\frac{1}{E} \frac{E^{p}\left(\varepsilon-\varepsilon^{p}\right)-E^{u}\left(\varepsilon-\varepsilon^{p}\right)\left(1-\frac{d \varepsilon^{p}}{d \varepsilon}\right)}{\left(\varepsilon-\varepsilon^{p}\right)^{2}}=-\frac{1}{E} \frac{E^{p}-E^{u}+E^{u} \frac{d \varepsilon^{p}}{d \varepsilon}}{\left(\varepsilon-\varepsilon^{p}\right)},
\end{aligned}
$$

и после подстановки данного выражения и формулы для $\omega$ в уравнение (11) получаем тождество. Аналогично данные выражения при $E^{u}=E^{s}, \varepsilon^{p}=0$ удовлетворяют уравнению (16).

Слагаемые в выражении для некомпенсированного тепла должны быть положительными величинами, т. е. $d g^{p} \geqslant 0, d g^{\omega} \geqslant 0$. Тогда, подставляя выражение (8) в фрормулу (6), имеем

$$
d g^{\omega}=\frac{1}{2} E \varepsilon^{e} \varepsilon^{e}\left(-\frac{d E^{u}}{E}\right) \geqslant 0
$$

и, следовательно, $d E^{u} \leqslant 0$. Если в выражение для $d \varepsilon^{p}$ из формулы (12) подставить в формулу (3), то получим

$$
d g^{p}=\sigma d \varepsilon-\sigma \frac{E^{p}}{E^{u}} d \varepsilon+\frac{\sigma\left(\varepsilon-\varepsilon^{p}\right)}{E^{u}} d E^{u} \geqslant 0 .
$$

Используя равенства (10) и (5), находим

$$
d g^{p}=\frac{\sigma d \sigma}{E^{p}}-\frac{\sigma d \sigma}{E^{u}}+\frac{\sigma^{2}}{\left(E^{u}\right)^{2}} d E^{u} \geqslant 0 .
$$

Так как $d E^{u} \leqslant 0$, то данное неравенство справедливо тогда, когда

$$
\sigma d \sigma\left(\frac{1}{E^{p}}-\frac{1}{E^{u}}\right)>0
$$

Отсюда следует, что $E^{u}>E^{p}$. Тогда неравенство (18) выполняется как на стадии упрочнения $\left(d \sigma>0, E^{p}>0\right)$, так и на стадии разупрочнения $\left(d \sigma<0, E^{p}<0\right)$. Теперь из выражения (17) с учётом неравенства (18) получаем неравенство

$$
\left|d E^{u}\right| \leqslant\left(\frac{E^{u}}{E^{p}}-1\right) E^{u} \frac{d \sigma}{\sigma} .
$$

Условие (19) накладывает ограничение на скорость убывания модуля разгрузки, которое обусловлено континуальным разрушением. Оно обеспечивает выполнение неравенства $d \varepsilon^{p} \geqslant 0$ и, следовательно, условия $d g^{p} \geqslant 0$. 
Если $\omega=0$ (упругопластический материал), то с учётом равенства (14) имеем

$$
d g^{p}=\sigma\left(1-\frac{E^{p}}{E}\right) d \varepsilon=\sigma\left(1-\frac{E^{p}}{E}\right) \frac{d \sigma}{E^{p}}, \quad d g^{\omega}=0 .
$$

Величина $d g^{p}$ положительна как на стадии упрочнения ( $\left.E^{p}>0, d \sigma>0\right)$, так и на стадии разупрочнения $\left(E^{p}<0, d \sigma<0\right)$. Для упруго хрупкого материала $d g^{p}=0, d g^{\omega}=\frac{1}{2} E \varepsilon \varepsilon\left(-\frac{d E^{s}}{E}\right)$ и необходимо $d E^{s} \leqslant 0$, что действительно выполняется.

Таким образом, модель упругопластического материала с разупрочнением, модель партипластического материала при условия $d E^{u} \leqslant 0$ и (19) и модель упруго хрупкого материала не противоречат законам термодинамики.

Далее естественно полагать, что материал разрушен, если он потерял способность к релаксации напряжений или, другими словами, не может диссипировать подводимую извне энергию, то есть $d g^{p}=0, d g^{\omega}=0$. Отсюда, когда $\omega=0, d \varepsilon^{p}=0$ (релаксация только за счёт пластической деформации), то, приравнивая выражение (3) к нулю, получаем при разрушении $\sigma=0$. Из этого условия и соотношения (13) вытекает также равенство $\varepsilon=\varepsilon^{p}$ или $d \varepsilon=d \varepsilon^{p}\left(E^{p}=0\right)$. Следовательно, после разрушения пластическая деформация совпадает с полной деформацией, а упругая составляющая отсутствует.

Если $\varepsilon^{p}=0$ (релаксация только за счёт микроразрушений), то, приравнивая выражение (6) к нулю, имеем $d \omega=0$ или $\omega=$ const, то есть повреждённость материала при разрушении достигает постоянного значения. Из равенства (8), в котором в данном случае $E^{u}=E^{s}$, вытекает, что после разрушения $d E^{\mathcal{s}}=0$, т.е. $E^{\mathcal{s}}=$ const. При возрастающей деформации это возможно только в одном случае, когда секущий модуль $E^{s}$ обращается в нуль. Тогда из равенства (7) следует, что при разрушении повреждённость $\omega=1$. Кроме того, из соотношений (10) и (15) имеем $\sigma=0$, $d \sigma=0$ и $E^{p}=0$.

При разрушении партипластического материала возможны следующие варианты. В первом случае ещё до разрушения повреждённость достигает значения $\omega=1-\frac{E^{u}}{E}<1$ (разрушаются все хрупкие элементы). В дальнейшем $d \omega=0$ и, следовательно, диссипация $d g^{\omega}=0$. Разрушение материала происходит, когда $\sigma=0\left(d g^{p}=0\right)$ и $\varepsilon=\varepsilon^{p}, d \varepsilon=d \varepsilon^{p}, E^{p}=0$. Во втором случае ещё до разрушения исчерпывается пластичность материала, т. е. $d \varepsilon^{p}=0$ ( $\varepsilon^{p}=\varepsilon_{\max }^{p}=$ const). Затем диссипация идёт только за счёт континуального разрушения. Разделение материала на фрагменты наступает тогда, когда $d \omega=0$, т. е. повреждаемость достигает постоянного значения, равного единице. В этот момент $E^{u}=0, \sigma=0, d \sigma=0, E^{p}=0$. В третьем случае оба процесса диссипации продолжаются вплоть до разрушения. Так как модуль разгрузки уменьшается до нуля (рис. 3, в), то повреждённость $\omega$ достигает значения равного единице. Тогда $d \omega=0$ и $d g^{\omega}=0$. Из соотношения (5) находим, что в момент разрушения $\sigma=0$ и, следовательно, $d g^{p}=0$. Отметим, что в момент разрушения $\varepsilon-\varepsilon^{p} \neq 0$, так как в противном случае диссипация проходила бы только за счёт пластической деформации.

Далее уравнение (11) можно переписать в виде

$$
d \varepsilon_{\omega}^{e}=d \varepsilon_{\omega}^{p}-d \varepsilon^{p}
$$

где $d \varepsilon_{\omega}^{e}=d\left(\omega\left(\varepsilon-\varepsilon^{p}\right)\right)$

$$
d \varepsilon_{\omega}^{p}=\left(1-\frac{E^{p}}{E}\right) d \varepsilon
$$

Здесь $d \varepsilon_{\omega}^{p}$ - приращение псевдопластической деформации, которое могло бы иметь место, если бы модуль разгрузки был равен $E$. Аналогичное представление имеет и уравнение (16), а именно, $d \varepsilon_{\omega}=d \varepsilon_{\omega}^{p}$, где $\varepsilon_{\omega}=\omega \varepsilon$.

Вернемся опять к уравнению (5) и запишем его в виде

$$
\sigma=E\left[\varepsilon-\varepsilon^{p}-\omega\left(\varepsilon-\varepsilon^{p}\right)\right]=E\left[\varepsilon-\left(\varepsilon^{p}+\varepsilon_{\omega}^{e}\right)\right]=E\left(\varepsilon-\varepsilon_{\omega}^{p}\right),
$$

где $\varepsilon_{\omega}^{p}$ - величина полной псевдопластической деформации, которую можно найти, если считать, что модуль разгрузки равен $E$. Кинетика её развития зависит от изменения $\varepsilon^{p}$ и $\omega$. С формальной точки зрения она определяется уравнением (20). Отметим, что соотношением (21) и законом (20) можно пользоваться, если имеет место активный процесс деформирования (без разгрузок). 
И, наконец, выпишем определяющие соотношения при разгрузке и последующем сжатии в области упругости. Для упруго хрупкого материала при разгрузке имеем

$$
\sigma=\sigma^{u}-E^{s}\left(\varepsilon^{u}\right)\left(\varepsilon^{u}-\varepsilon\right), \quad 0 \leqslant \varepsilon \leqslant \varepsilon^{u},
$$

при сжатии -

$$
\sigma=E \varepsilon, \quad \varepsilon<0
$$

для упругопластического материала -

$$
\sigma=\sigma^{u}-E\left(\varepsilon^{u}-\varepsilon\right), \quad \varepsilon \leqslant \varepsilon^{u} .
$$

Для партипластического материала имеем при разгрузке

$$
\sigma=\sigma^{u}-E^{s}\left(\varepsilon^{u}\right)\left(\varepsilon^{u}-\varepsilon\right), \quad \varepsilon^{p}\left(\varepsilon^{u}\right) \leqslant \varepsilon \leqslant \varepsilon^{u},
$$

при сжатии -

$$
\sigma=E_{b}^{u}\left(\varepsilon-\varepsilon^{p}\left(\varepsilon^{u}\right)\right), \quad \varepsilon<\varepsilon^{p}\left(\varepsilon^{u}\right) .
$$

Здесь $\sigma^{u}, \varepsilon^{u}$ - соответственно напряжение и дефрормация в начале разгрузки, $\varepsilon^{p}\left(\varepsilon^{u}\right)-$ пластическая деформация, отвечающая деформации начала разгрузки.

3. Энергия упругих деформаций и диссипация. Потенциальная энергия упругих деформаций для упруго хрупкого материала определяется выражением $\Pi^{e}=\frac{1}{2} E(1-\omega) \varepsilon^{2}=\frac{1}{2} E^{s} \varepsilon^{2}$. Её приращение равно

$$
d \Pi^{e}=E(1-\omega) \varepsilon d \varepsilon-\frac{1}{2} E \varepsilon^{2} d \omega=E^{s} \varepsilon d \varepsilon-d g^{\omega}=\sigma d \varepsilon-d g^{\omega} .
$$

Если $d \Pi^{e}>0$, то подводимая энергия превышает диссипацию; если $d \Pi^{e}<0$, то материал диссипирует всю подведённую энергию и ещё часть энергии, накопленной материалом.

Рассмотрим выражение

$$
d(\sigma \varepsilon)=\sigma d \varepsilon+\varepsilon d \sigma=\sigma d \varepsilon+\varepsilon E d[(1-\omega) \varepsilon]=\sigma d \varepsilon+E \varepsilon(1-\omega) d \varepsilon-\varepsilon E \varepsilon d \omega=2 \sigma d \varepsilon-2 d g^{\omega} .
$$

Отсюда $d g^{\omega}=\frac{1}{2}(\sigma d \varepsilon-\varepsilon d \sigma)$. Сравнивая далее формулы (22) и (23), находим, что $d \Pi^{e}=\frac{1}{2} d(\sigma \varepsilon)$.

Далее, подставляя выражение повреждённости (7), где $E^{u}=E^{s}$, в формулу для потенциальной энергии, имеем

$$
d \Pi^{e}=E^{s} \varepsilon d \varepsilon+\frac{1}{2} \varepsilon^{2} d E^{s}=E^{s} \varepsilon d \varepsilon+\frac{1}{2} \varepsilon\left(E^{p}-E^{s}\right) d \varepsilon=\frac{1}{2}\left(E^{s}+E^{p}\right) \varepsilon d \varepsilon
$$

Здесь

$$
\frac{d E^{s}}{d \varepsilon}=\frac{d}{d \varepsilon}\left(\frac{\sigma}{\varepsilon}\right)=\frac{1}{\varepsilon^{2}}\left(\frac{d \sigma}{d \varepsilon} \varepsilon-\sigma\right)=\frac{1}{\varepsilon}\left(E^{p}-E^{s}\right) .
$$

Из выражения (24) следует, что $d \Pi^{e}>0$, когда $E^{s}+E^{p}>0$, и $d \Pi^{e}<0$, когда $E^{s}+E^{p}<0$. Отсюда диссипация начинает превышать подводимую энергию, если $E^{p}<-E^{s}\left(E^{\mathcal{s}}>0\right)$. Следовательно, после выхода на падающую ветвь диаграммы $\sigma(\varepsilon)$, несмотря на падение напряжения при росте деформации, упруго хрупкий материал некоторое время продолжает накапливать потенциальную энергию упругих деформаций.

Найдём теперь величину энергии $\Pi^{\omega}$, затраченной на континуальное разрушение. С учётом выражения (25) имеем

$$
d g^{\omega}=-\frac{1}{2} \varepsilon^{2} d E^{s}=-\varepsilon\left(E^{p}-E^{s}\right) d \varepsilon=\frac{\sigma}{E^{s}}\left(E^{s}-E^{p}\right) d \varepsilon .
$$

Тогда при достижении деформацией некоторой величины $\varepsilon$ находим

$$
\Pi^{\omega}(\varepsilon)=g^{\omega}(\varepsilon)=\int_{\varepsilon^{t}}^{\varepsilon} d g^{\omega}=-\frac{1}{2} \int_{\varepsilon^{t}}^{\varepsilon} \sigma E^{p}\left(E^{s}\right)^{-1} d \varepsilon=I_{1}-\frac{1}{2} I_{2}
$$


(диссипация до предела пропорциональности равна нулю). Интегрируя по частям второй интеграл в формуле (26), получаем

$$
I_{2}=\int_{\varepsilon^{t}}^{\varepsilon} E^{p} \varepsilon d \varepsilon=\int_{\varepsilon^{t}}^{\varepsilon} \frac{d \sigma}{d \varepsilon} \varepsilon d \varepsilon=\int_{\sigma^{t}}^{\sigma} \varepsilon d \sigma=\left.\varepsilon \sigma\right|_{\sigma^{t}} ^{\sigma}-\int_{\varepsilon^{t}}^{\varepsilon} \sigma d \varepsilon=\varepsilon \sigma-\varepsilon^{t} \sigma^{t}-2 I_{1}
$$

Здесь $\sigma^{t}=E \varepsilon^{t}$. Итак

$$
\Pi^{\omega}(\varepsilon)=\int_{\varepsilon^{t}}^{\varepsilon} \sigma d \varepsilon+\frac{1}{2} \varepsilon^{t} \sigma^{t}-\frac{1}{2} \sigma \varepsilon
$$

Очевидно, что данное значение функции $\Pi^{\omega}(\varepsilon)$ численно равно площади, ограниченной диаграммой деформирования и прямой, определяемой секущим модулем $E^{\mathcal{s}}(\varepsilon)$.

Энергия упругих деформаций, накопленная материалом, равна $\Pi^{e}=\frac{1}{2} \sigma \varepsilon$. Тогда величина полной энергии, затраченной на деформацию, определяется выражением

$$
\Pi=\Pi^{e}+\Pi^{\omega}=\int_{\varepsilon^{t}}^{\varepsilon} \sigma d \varepsilon+\frac{1}{2} \varepsilon^{t} \sigma^{t}=\int_{0}^{\varepsilon} \sigma d \varepsilon
$$

Её численное значение совпадает с площадью, ограниченной диаграммой деформирования.

При разрушении $\left(\varepsilon=\varepsilon^{z}, \sigma=0\right)$ имеем $\Pi^{e}=0, \Pi^{\omega}=\Pi$. Следовательно, в этот момент диссипирована вся затраченная на деформацию энергия, которая равна площади, ограниченной всей диаграммой деформирования.

Потенциальная энергия упругих деформаций для упругопластического материала определяется формулой $\Pi^{e}=\frac{1}{2} E \varepsilon^{e} \varepsilon^{e}=\frac{1}{2} E\left(\varepsilon-\varepsilon^{p}\right)^{2}$. Приращение этой энергии определяется выражением

$$
\Pi^{e}=E\left(\varepsilon-\varepsilon^{p}\right)\left(d \varepsilon-d \varepsilon^{p}\right)=\sigma\left(d \varepsilon-d \varepsilon^{p}\right)=\sigma d \varepsilon-\sigma d \varepsilon^{p}=\sigma d \varepsilon-d g^{p} .
$$

С учётом закона пластичности (14) находим, что

$$
d \Pi^{e}=\sigma d \varepsilon-\sigma\left(1-\frac{E^{p}}{E}\right) d \varepsilon=\frac{E^{p}}{E} \sigma d \varepsilon .
$$

Отсюда $d \Pi^{e}>0$ (диссипируется меньше энергии, чем подводится) при $E^{p}>0$ и $d \Pi^{e}<0$ при $E^{p}<0$. Таким образом, диссипация начинает превышать подводимую энергию сразу после выхода на падающую ветвь диаграммы деформирования. При этом $d \varepsilon^{p}>d \varepsilon$, т. е. приращение пластической деформации превышает приращение полной деформации.

Величина энергии, затраченной на пластическую деформацию, равна

$$
\Pi^{p}(\varepsilon)=g^{p}(\varepsilon)=\int_{\varepsilon^{T}}^{\varepsilon} \sigma d \varepsilon^{p}=\int_{\varepsilon^{T}}^{\varepsilon} \sigma\left(1-\frac{E^{p}}{E}\right) d \varepsilon=\int_{\varepsilon^{T}}^{\varepsilon} \sigma d \varepsilon-\frac{1}{E} \int_{\varepsilon^{T}}^{\varepsilon} \sigma E^{p} d \varepsilon=\int_{\varepsilon^{T}}^{\varepsilon} \sigma d \varepsilon-\frac{1}{E} \int_{\varepsilon^{T}}^{\varepsilon} \sigma d \sigma=\int_{\varepsilon^{T}}^{\varepsilon} \sigma d \varepsilon+\frac{\sigma^{T} \varepsilon^{T}}{2}-\frac{1}{2} \frac{\sigma^{2}}{E} .
$$

Таким образом, эта величина равняется площади, ограниченной диаграммой деформирования и прямой, определяемой модулем разгрузки, равным $E$.

Выражение для энергии упругих деформаций можно записать в виде $\Pi^{e}=\frac{1}{2} \frac{\sigma^{2}}{E}$. Тогда

$$
\Pi=\Pi^{e}+\Pi^{p}=\int_{\varepsilon^{T}}^{\varepsilon} \sigma d \varepsilon+\frac{\sigma^{T} \varepsilon^{T}}{2}=\int_{0}^{\varepsilon} \sigma d \varepsilon,
$$

т. е. полная энергия, затраченная на деформацию, численно равна площади, ограниченной диаграммой деформирования.

При разрушении имеем $\Pi^{e}=0, \Pi^{p}=\Pi$ и вся затраченная на дефрормирования материала энергия ушла на образование пластической деформации.

Приведённые выше рассуждения позволяют ввести две характеристики упругопластического материала - меру исчерпания пластичности $\Phi=\frac{g^{p}(\varepsilon)}{g^{p}\left(\varepsilon^{2}\right)}$ и меру ресурса пластичности $\Psi=1-\Phi$. 
Отметим наконец, что в модели упругопластического материала при активном (без разгрузок) деформировании имеется три потенциала напряжений: первый - это полная энергия (как в случае упруго хрупкого материала), т. е. $\sigma(\varepsilon)=\frac{d \Pi}{d \varepsilon}$, второй - это энергия диссипации $\Pi^{p}$, т. е. $\sigma\left(\varepsilon^{p}\right)=\frac{d \Pi^{p}}{d \varepsilon^{p}}$, и третий - энергия упругих деформаций $\Pi^{e}$, т. е $\sigma\left(\varepsilon^{e}\right)=\frac{d \Pi^{e}}{d \varepsilon^{e}}$. График диаграммы диссипации $\sigma\left(\varepsilon^{p}\right)$ показан на рис. 5. Площадь, ограниченная ею при текущем значении пластической деформации, равна текущей диссипации (заштрихованная область на рис. 5). Касательная к данной диаграмме является инкрементальным модулем упрочнения (разупрочнения) Н. Отсюда

$$
H=\frac{d \sigma}{d \varepsilon^{p}}=\frac{d^{2} \Pi^{p}}{\left(d \varepsilon^{p}\right)^{2}}
$$

и $d \sigma=H d \varepsilon^{p}$. Подставляя в данное выражение значение $d \varepsilon^{p}$ из закона (14) и, используя равенство (10), находим, что

$$
H=\frac{E E^{p}}{\left(E-E^{p}\right)} .
$$

Следовательно, на стадии упрочнения $H>0\left(E^{p}>0, E-E^{p}>0\right)$, а на стадии разупрочнения $H<0$ $\left(E^{p}<0, E-E^{p}>0\right)$.

Для партипластического материала потенциальная энергия упругих деформаций определяется выражением $\Pi^{e}=\frac{1}{2} E(1-\omega)\left(\varepsilon-\varepsilon^{p}\right)^{2}$. Тогда

$$
d \Pi^{e}=E(1-\omega)\left(\varepsilon-\varepsilon^{p}\right)\left(d \varepsilon-d \varepsilon^{p}\right)-\frac{1}{2} E\left(\varepsilon-\varepsilon^{p}\right)^{2} d \omega=\sigma\left(d \varepsilon-d \varepsilon^{p}\right)-\frac{1}{2} E \varepsilon^{e} \varepsilon^{e} d \omega=\sigma d \varepsilon-d g^{\omega}-d g^{p} .
$$

С учётом выражений для приращения диссипаций (17) и (6), где в последнем $\varepsilon^{e}=\varepsilon-\varepsilon^{p}=\frac{\sigma}{E^{u}}$, $d \omega=-\frac{d E^{u}}{E}$, после простых преобразований находим

$$
d \Pi^{e}=\left(E^{p}-\frac{1}{2} \frac{\sigma}{E^{u}} \frac{d E^{u}}{d \varepsilon}\right) \frac{\sigma d \varepsilon}{E^{u}} .
$$

На восходящей ветви диаграммы деформирования $d \Pi^{e}>0\left(E^{u}>0, E^{p}>0, \sigma>0, \frac{d E^{u}}{d \varepsilon}<0\right)$. На падающей ветви энергия упругих деформаций сначала продолжает расти, а с момента, когда

$$
E^{p}<\frac{1}{2} \frac{\sigma}{E^{u}} \frac{d E^{u}}{d \varepsilon}
$$

начинает убывать $\left(d \Pi^{e}<0\right)$.

Механическая диссипация (энергия, затраченная на образование пластических деформаций) с учётом выражения (17) равна

$$
\Pi^{p}(\varepsilon)=g^{p}(\varepsilon)=\int_{\varepsilon^{t}}^{\varepsilon} d g^{p}=\int_{\varepsilon^{t}}^{\varepsilon} \sigma d \varepsilon-\int_{\varepsilon^{t}}^{\varepsilon} \frac{\sigma d \sigma}{E^{u}}+\int_{\varepsilon^{t}}^{\varepsilon}\left(\frac{\sigma}{E^{u}}\right)^{2} d E^{u} .
$$

Интегрируя по частям второй интеграл, имеем

$$
\int_{\varepsilon^{t}}^{\varepsilon} \frac{\sigma d \sigma}{E^{u}}=\left.\frac{1}{2} \frac{\sigma^{2}}{E^{u}}\right|_{\sigma^{t}} ^{\sigma}+\frac{1}{2} \int_{\varepsilon^{t}}^{\varepsilon}\left(\frac{\sigma}{E^{u}}\right)^{2} \frac{d E^{u}}{d \varepsilon} d \varepsilon
$$

Тогда

$$
\Pi^{p}(\varepsilon)=\int_{\varepsilon^{t}}^{\varepsilon} \sigma d \varepsilon-\frac{1}{2} \frac{\sigma^{2}}{E^{u}}+\frac{1}{2} \sigma^{t} \varepsilon^{t}+\frac{1}{2} \int_{\varepsilon^{t}}^{\varepsilon}\left(\frac{\sigma}{E^{u}}\right)^{2} d E^{u} .
$$


Энергия, затраченная на континуальное разрушение, равна

$$
\Pi^{\omega}(\varepsilon)=g^{\omega}(\varepsilon)=\int_{\varepsilon^{t}}^{\varepsilon} d g^{\omega}=-\frac{1}{2} \int_{\varepsilon^{t}}^{\varepsilon} \varepsilon^{e} \varepsilon^{e} d E^{u}=-\frac{1}{2} \int_{\varepsilon^{t}}^{\varepsilon}\left(\frac{\sigma}{E^{u}}\right)^{2} d E^{u} .
$$

Отсюда энергия диссипации определяется выражением

$$
g(\varepsilon)=\int_{\varepsilon^{t}}^{\varepsilon} \sigma d \varepsilon+\frac{1}{2} \sigma^{t} \varepsilon^{t}-\frac{\sigma^{2}}{2 E^{u}}=\int_{0}^{\varepsilon} \sigma d \varepsilon-\Pi^{e} .
$$

Таким образом, текущая диссипация равна площади, ограниченной диаграммой деформирования и прямой разгрузки, а полная диссипация (диссипация разрушения) равна площади под полной диаграммой деформирования.

Работа выполнена при финансовой поддержке Российского фбонда фбундаментальнъх исследований (проект № 07-01-96087)

\section{БИБЛИОГРАФИЧЕСКИЙ СПИСОК}

1. Волков, С.Д. Экспериментальные функции сопротивления легированной стали при растяжении с кручением [Текст] / С. Д. Волков, Ю. П. Гуськов, В. И. Кривоспицкая и др. // Проблемы прочности. $-1979 .-$ Т. 11, № 1. C. $3-6$.

2. Лебедев, А.А. Кинетика разрушения листовой аустенитной стали на заключительной стадии деформирования [Текст] / А. А. Лебедев, Н. Г. Чаусов, О.И.Марусий // Проблемы прочности. - 1989. - № 3. - С. 16-21.

3. Стружанов, В.В. Модифицированная модель Мазинга [Текст] / В. В. Стружанов, В. В. Башуров // Вестн. Сам. госуд. техн. ун-та. Сер.: Физ.-мат. науки. - 2007. - № 1 (14). - С. 29-39. - ISSN 1991-8615.

4. Радченко, В.П. Структурно-феноменологический подход к описанию полной диаграммы упругопластического деформирования [Текст] / В. П. Радченко, Е. В. Небогина, М. В. Басов // Изв. вузов. Машиностроение. - 2000. № 5-6. - C. 3-13. - ISSN 0536-1044.

5. Качанов, Л.М. Основы теории пластичности [Текст] / Л. М. Качанов. - М.: Наука, 1969. - 420 с.

6. Жуков, А.М. Некоторые особенности поведения металлов при упругопластическом деформировании [Текст] / А. М. Жуков / В сб.: Вопросы теории пластичности. - Изд-во АН СССР, 1961. - С. 30-57.

7. Работнов, Ю.Н. Механика деформируемого твёрдого тела [Текст] / Ю. Н. Работнов. - М.: Наука, $1988 .-712$ с.

8. Седов, Л.И. Механика сплошной среды [Текст] / Л. И. Седов. - М.: Наука, 1976. - Т. 2. - 576 с.

Институт машиноведения Уральского отделения РАН, г. Екатеринбург

Поступила 01.06.2007 